\title{
ON SOME CONTINUED FRACTION IDENTITIES OF SRINIVASA RAMANUJAN ${ }^{1}$
}

\section{S. BHARGAVA AND CHANDRASHEKAR ADIGA}

\begin{abstract}
The main purpose of this note is to state and prove, in a simple, unified manner, several $q$-continued fraction expansions found in Ramanujan's "lost" notebook. This is related to some recent works of G. E. Andrews and M. D. Hirschhorn.
\end{abstract}

0. Introduction. The following continued fraction identities $(1)_{R}-(3)_{R}$ and $(I)_{R}$ found in the "lost" notebook of Ramanujan (terminology due to G. E. Andrews [1]) contain as special cases many of his other identities:

$(1)_{\mathrm{R}}$

$$
\begin{aligned}
\frac{G(0, \lambda q, b, q)}{G(0, \lambda, b, q)}= & \frac{1}{1}+\frac{\lambda q}{1}+\frac{b q+\lambda q^{2}}{1} \\
& +\cdots \frac{\lambda q^{2 n+1}}{1}+\frac{b q^{n+1}+\lambda q^{2 n+2}}{1}+\cdots \\
= & \frac{1}{1}+\frac{\lambda q}{1+b q}+\frac{\lambda q^{2}}{1+b q^{2}}+\cdots \frac{\lambda q^{n}}{1+b q^{n}}+\cdots \\
= & \frac{1}{1-b}+\frac{b+\lambda q}{1-b}+\cdots \frac{b+\lambda q^{n}}{1-b}+\cdots
\end{aligned}
$$

and, more generally

$$
\begin{aligned}
& (\mathrm{I})_{\mathrm{R}} \quad \frac{G(a q, \lambda q, b, q)}{G(a, \lambda, b, q)}=\frac{1}{1}+\frac{a q+\lambda q}{1}+\frac{b q+\lambda q^{2}}{1} \\
& +\cdots \frac{a q^{n+1}+\lambda q^{2 n+1}}{1}+\frac{b q^{n+1}+\lambda q^{2 n+2}}{1}+\cdots
\end{aligned}
$$

where

$$
G(a, \lambda, b, q)=\sum_{n=0}^{\infty} \frac{q^{n(n+1) / 2}(-\lambda / a)_{n} a^{n}}{(q)_{n}(-b q)_{n}} .
$$

Here and in what follows,

$$
(c)_{k}=\left\{\begin{array}{l}
1 \quad \text { if } k=0, \\
(1-c)(1-c q) \cdots\left(1-c q^{k-1}\right) \quad \text { if } k>0 .
\end{array}\right.
$$

Received by the editors July $25,1983$.

1980 Mathematics Subject Classification. Primary 10A32, 10A35, 30B70, 40A15.

Key words and phrases. q-continued fractions, Rogers-Ramanujan identifies.

${ }^{1}$ A summary of part of this paper was presented by the authors at the 48th conference of the Indian Mathematical Society held at Bhagalpur, India on December 26-28, 1982. 
The suffix $R$ signifies that the identity is due to Ramanujan. It is easily seen that $(1)_{R}-(3)_{R}$ are themselves special cases, respectively, of $(\mathrm{I})_{R}$ above and (II) and (III) ${ }_{H}$ below:

$(\mathrm{III})_{\mathrm{H}}$

$$
\begin{gathered}
\frac{G(a q, \lambda q, b, q)}{G(a, \lambda, b, q)}=\frac{1}{1}+\frac{a q+\lambda q}{1-a q+b q}+\cdots \frac{a q+\lambda q^{n}}{1-a q+b q^{n}}+\cdots \\
\quad=\frac{1}{1-b+a q}+\frac{b+\lambda q}{1-b+a q^{2}}+\cdots \frac{b+\lambda q^{n}}{1-b+a q^{n+1}}+\cdots
\end{gathered}
$$

The suffix $\mathrm{H}$ in (III) $)_{\mathrm{H}}$ signifies that the identity is due to M. D. Hirschhorn [3]. Identity $(\mathrm{I})_{\mathrm{R}}$, and thereby $(1)_{\mathrm{R}}$, has been proved independently by Andrews [1] and by Hirschhorn [4]. Andrews has employed $G$ and some auxiliary functions and a transformation of E. Heine; and Hirschhorn has proved it by obtaining a closed form for the $n$th convergent. While Andrews [2] has given a separate proof of the "slightly tricky" identity $(2)_{R}$, he has extracted $(3)_{R}$ as a particular case of (III) ${ }_{H}$ which Hirschhorn [3] has proved by finding a closed form for the $n$th convergent. Many other identities of Ramanujan also follow as pointed out by Andrews and Hirschhorn. However, we have not come across (II) nor a proof of the following Ramanujan identities (IV) ${ }_{\mathrm{R}}$ and (5) $)_{\mathrm{R}}$ also listed in the "lost" notebook:

$$
\begin{aligned}
\frac{G(a q, \lambda q, b, q)}{G(a, \lambda, b, q)}= & \frac{1}{1+a q}+\frac{\lambda q-a b q^{2}}{1+q(a q+b)} \\
& +\cdots \frac{\lambda q^{n}-a b q^{2 n}}{1+q^{n}(a q+b)}+\cdots
\end{aligned}
$$

$(5)_{\mathrm{R}} \frac{1}{a+c}-\frac{a b}{a+b+c q}-\cdots \frac{a b}{a+b+c q^{n}}-\ldots$

$$
=\frac{1}{c-b+a}+\frac{b c}{c-b+a / q}+\cdots \frac{b c}{c-b+a / q^{n}}+\cdots \cdot
$$

In what follows we employ, as auxiliary function instead of $G(a, \lambda, b, q)$ a multiple of it, namely,

$$
g(a, \lambda, b, q)=(-b q)_{\infty} \sum_{n=0}^{\infty} \frac{q^{n(n+1) / 2}(-\lambda / a)_{n} a^{n}}{(q)_{n}(-b q)_{n}}
$$

and will thereby be able to give a simple, unified and self-contained approach to proving $(\mathrm{I})_{\mathrm{R}},(\mathrm{II}),(\mathrm{III})_{\mathrm{H}},(\mathrm{IV})_{\mathrm{R}}$ and $(5)_{\mathrm{R}}$. We may observe that in all the identities (I)-(IV) and in $(1)_{R}-(3)_{R}$ we may replace $G$ by $g$. We deduce (I)-(IV) directly $(\S \S 2-5)$ from three easily proved canonical functional relations $(6)-(8)$ for $g(\S 1)$ and extract $(5)_{R}(\S 6)$ as a particular case of the identity (II) $=(I V)_{R}$ with $\lambda=0$.

\section{Three canonical functional relations satisfied by $g$.}

LEMMA 1 (KEY LEMMA). If $|q|<1$, then $g$ satisfies the following functional relations:

$$
\begin{aligned}
& g(a, \lambda, b, q)-g(a q, \lambda, b, q)=a q g(a q, \lambda q, b q, q), \\
& g(a, \lambda, b, q)-g(a, \lambda q, b, q)=\lambda q g\left(a q, \lambda q^{2}, b q, q\right), \\
& g(a, \lambda, b, q)-g(a, \lambda, b q, q)=b q g(a q, \lambda q, b q, q) .
\end{aligned}
$$


Proof. Since,

$$
(-\lambda / a)_{n}-q^{n}(-\lambda / a q)_{n}=\left\{\begin{array}{l}
0 \quad \text { if } n=0, \\
(-\lambda / a)_{n-1}\left(1-q^{n}\right) \text { if } n>0,
\end{array}\right.
$$

as easily verified, we have

$$
\text { left side of } \begin{aligned}
(6) & =(-b q)_{\infty} \sum_{n=0}^{\infty} \frac{q^{n(n+1) / 2} a^{n}}{(q)_{n}(-b q)_{n}}\left\{\left(-\frac{\lambda}{a}\right)_{n}-q^{n}\left(-\frac{\lambda}{a q}\right)_{n}\right\} \\
& =a q\left(-b q^{2}\right)_{\infty} \sum_{n=1}^{\infty} \frac{q^{n(n-1) / 2}(a q)^{n-1}(-\lambda / a)_{n-1}}{(q)_{n-1}\left(-b q^{2}\right)_{n-1}} \\
& =a q g(a q, \lambda q, b q, q), \text { proving (6). }
\end{aligned}
$$

In the penultimate step we have used the obvious identity

$$
\frac{(-b q)_{\infty}}{(-b q)_{n}}=\frac{\left(-b q^{2}\right)_{\infty}}{\left(-b q^{2}\right)_{n-1}}
$$

Relations (7) and (8) follow in exactly the same way using

$$
(-\lambda / a)_{n}-(-\lambda q / a)_{n}=\left\{\begin{array}{l}
0 \text { if } n=0, \\
\frac{\lambda}{a}(-\lambda q / a)_{n-1}\left(1-q^{n}\right) \text { if } n>0,
\end{array}\right.
$$

and

$$
\frac{(-b q)_{\infty}}{(-b q)_{n}}-\frac{\left(-b q^{2}\right)_{\infty}}{\left(-b q^{2}\right)_{n}}=\frac{\left(-b q^{2}\right)_{\infty}}{\left(-b q^{2}\right)_{n}} b q^{n+1}
$$

Lemmas 2-5 proved below are simple combinations of relations (6)-(8). Also, Theorems 1-4 follow directly from Lemmas 2-5, respectively, in a simple manner.

\section{Proof of the Ramanujan identity $(I)_{R}$.}

LEMMA 2. $g$ satisfies

$$
\begin{aligned}
& g(a, \lambda, b, q)=g(a q, \lambda q, b, q)+(a q+\lambda q) g\left(a q, \lambda q^{2}, b q, q\right), \\
& g(a, \lambda, b, q)=g(a, \lambda q, b q, q)+(b q+\lambda q) g\left(a q, \lambda q^{2}, b q, q\right) .
\end{aligned}
$$

Proof. Changing $\lambda$ to $\lambda q$ in (6) and adding to (7) gives (9), while changing $\lambda$ to $\lambda q$ in (8) and adding to (7) gives (10).

TheOREM 1. If $|q|<1$, then

$(\mathrm{I})_{\mathrm{R}}$

$$
\begin{aligned}
\rho= & \frac{1}{1}+\frac{a q+\lambda q}{1}+\frac{b q+\lambda q^{2}}{1} \\
& +\cdots \frac{a q^{n+1}+\lambda q^{2 n+1}}{1}+\frac{b q^{n+1}+\lambda q^{2 n+2}}{1}+\cdots
\end{aligned}
$$

where

$$
\rho=\frac{G(a q, \lambda q, b, q)}{G(a, \lambda, b, q)}=\frac{g(a q, \lambda q, b, q)}{g(a, \lambda, b, q)} .
$$


Proof. Changing $a$ to $a q^{n}, \lambda$ to $\lambda q^{2 n}, b$ to $b q^{n}$ in (9) and changing $a$ to $a q^{n+1}, \lambda$ to $\lambda q^{2 n+1}$ and $b$ to $b q^{n}$ in (10) we can write (9) and (10), respectively, as

$$
\begin{aligned}
& Q_{n} \equiv \frac{g\left(a q^{n}, \lambda q^{2 n}, b q^{n}, q\right)}{g\left(a q^{n+1}, \lambda q^{2 n+1}, b q^{n}, q\right)}=1+\frac{a q^{n+1}+\lambda q^{2 n+1}}{Q_{n}^{\prime}}, \\
& Q_{n}^{\prime} \equiv \frac{g\left(a q^{n+1}, \lambda q^{2 n+1}, b q^{n}, q\right)}{g\left(a q^{n+1}, \lambda q^{2 n+2}, b q^{n+1}, q\right)}=1+\frac{b q^{n+1}+\lambda q^{2 n+2}}{Q_{n+1}} .
\end{aligned}
$$

Iterating the last two identities alternately with $n=0,1,2, \ldots$, we have $(\mathrm{I})_{\mathrm{R}}$. Convergence of the continued fraction follows since $Q_{n}, Q_{n}^{\prime} \rightarrow 1$ as $n \rightarrow \infty$ when $|q|<1$.

\section{Proof of identity (II).}

LEMMA 3. g satisfies

$$
\begin{aligned}
g(a, \lambda, b, q)= & g(a q, \lambda q, b, q)+(a q+\lambda q) g\left(a q, \lambda q^{2}, b q, q\right) \\
g(a q, \lambda, b, q)= & (1-a q+b q) g(a q, \lambda q, b q, q) \\
& +(a q+\lambda q) g\left(a q, \lambda q^{2}, b q^{2}, q\right)
\end{aligned}
$$

Proof. Changing $\lambda$ to $\lambda q$ in (6) and adding it to (7), we have (11). Changing $\lambda$ to $\lambda q$ and $b$ to $b q$ in (6), $b$ to $b q$ in (7), taking the negative of (6) and adding these three equalities to (8), we deduce (12).

THEOREM 2. If $|q|<1$, then

$$
\rho=\frac{1}{1}+\frac{a q+\lambda q}{1-a q+b q}+\cdots \frac{a q+\lambda q^{n}}{1-a q+b q^{n}}+\cdots
$$

where $\rho$ is as in Theorem 1.

Proof. (11) can be written as

$$
\frac{g(a q, \lambda q, b, q)}{g(a, \lambda, b, q)}=\frac{1}{1}+\frac{a q+\lambda q}{\frac{g(a q, \lambda q, b, q)}{g\left(a q, \lambda q^{2}, b q, q\right)}} .
$$

Changing $\lambda$ to $\lambda q^{n+1}$ and $b$ to $b q^{n}$, (12) can be written as

$$
S_{n} \equiv \frac{g\left(a q, \lambda q^{n+1}, b q^{n}, q\right)}{g\left(a q, \lambda q^{n+2}, b q^{n+1}, q\right)}=\left(1-a q+b q^{n+1}\right)+\frac{a q+\lambda q^{n+2}}{S_{n+1}}
$$

Iterating this with $n=0,1,2, \ldots$, and using (13) we have (II). Convergence of (II) follows since $S_{n} \rightarrow 1$ as $n \rightarrow \infty$ when $|q|<1$.

\section{Proof of identity (III) $)_{\mathrm{H}}$ of Hirschhorn.}

LEMMA 4. g satisfies

$$
\begin{aligned}
g(a, \lambda, b q, q)= & (1-b q+a q) g(a q, \lambda q, b q, q) \\
& +(b q+\lambda q) g\left(a q^{2}, \lambda q^{2}, b q, q\right)
\end{aligned}
$$


Proof. Changing $a$ to $a q$ in (7), $a$ to $a q$ and $\lambda$ to $\lambda q$ in (8), taking the negative of (8) and adding these three equalities to (6), we deduce (14).

THEOREM 3. If $|q|<1$, then

$(\text { III })_{\mathrm{H}} \quad \rho=\frac{1}{1-b+a q}+\frac{b+\lambda q}{1-b+a q^{2}}+\cdots \frac{b+\lambda q^{n}}{1-b+a q^{n+1}}+\cdots$

where $\rho$ is as in Theorems 1 and 2.

Proof. Changing $a$ to $a q^{n}, \lambda$ to $\lambda q^{n}$ and $b$ to $b / q$, (14) can be written as

$$
T_{n} \equiv \frac{g\left(a q^{n}, \lambda q^{n}, b, q\right)}{g\left(a q^{n+1}, \lambda q^{n+1}, b, q\right)}=\left(1-b+a q^{n+1}\right)+\frac{b+\lambda q^{n+1}}{T_{n+1}} .
$$

Iterating this with $n=0,1,2, \ldots$, we have (III) $)_{\mathrm{H}}$. Convergence of (III) $)_{\mathrm{H}}$ follows as in the proof of Theorem 2 .

\section{Proof of the Ramanujan identity $(I V)_{R}$.}

LEMMA 5. g satisfies

$$
\begin{aligned}
g(a, \lambda, b q, q)= & (1+a q) g(a q, \lambda q, b q, q) \\
& +\left(\lambda q-a b q^{3}\right) g\left(a q^{2}, \lambda q^{2}, b q^{2}, q\right), \\
g(a q, \lambda q, b, q)= & \{1+q(a q+b)\} g\left(a q^{2}, \lambda q^{2}, b q, q\right) \\
& +\left(\lambda q^{2}-a b q^{4}\right) g\left(a q^{3}, \lambda q^{3}, b q^{2}, q\right) .
\end{aligned}
$$

Proof. Change $a$ to $a q, \lambda$ to $\lambda q, b$ to $b q$ in (6) and multiply the result by $-b q$; change $a$ to $a q, b$ to $b q$ in (7); change $a$ to $a q$ in (8); take the negative of (8) and add all these to (6) to obtain (15). Change $a$ to $a q, \lambda$ to $\lambda q$ in (15); change $a$ to $a q$ and $\lambda$ to $\lambda q$ in (8) and add to get (16).

THEOREM 4. If $|q|<1$, then

$$
(\mathrm{IV})_{\mathrm{R}} \quad \rho=\frac{1}{1+a q}+\frac{\lambda q-a b q^{2}}{1+q(a q+b)}+\cdots \frac{\lambda q^{n}-a b q^{2 n}}{1+q^{n}(a q+b)}+\cdots
$$

where $\rho$ is as in Theorems 1-3.

Proof. Changing $b$ to $b / q,(15)$ can be written as

$$
\frac{g(a q, \lambda q, b, q)}{g(a, \lambda, b, q)}=\frac{1}{1+a q}+\frac{\lambda q-a b q^{2}}{\frac{g(a q, \lambda q, b, q)}{g\left(a q^{2}, \lambda q^{2}, b q, q\right)}} .
$$

Changing $a, \lambda$ and $b$ to $a q^{n-1}, \lambda q^{n-1}$ and $b q^{n-1}$, respectively, (16) can be written as

$$
U_{n} \equiv \frac{g\left(a q^{n}, \lambda q^{n}, b q^{n-1}, q\right)}{g\left(a q^{n+1}, \lambda q^{n+1}, b q^{n}, q\right)}=1+q^{n}(a q+b)+\frac{\lambda q^{n+1}-a b q^{2 n+2}}{U_{n+1}}
$$

Iterating (18) with $n=1,2, \ldots$, and using (17) we have $(I V)_{R}$. Convergence of $(I V)_{R}$ follows since $U_{n} \rightarrow 1$ as $n \rightarrow \infty$ when $|q|<1$. 
6. Proof of the Ramanujan identity $(5)_{R}$. The following theorem is a corollary to $\S \S 3$ and 5. In fact, it is a particular case of the identity (II) $=(I V)_{R}$.

THEOREM 5. If $|q|<1$, then

$(5)_{\mathrm{R}} \frac{1}{a+c}-\frac{a b}{a+b+c q}-\cdots \frac{a b}{a+b+c q^{n}}-\cdots$

$$
=\frac{1}{c-b+a}+\frac{b c}{c-b+a / q}+\cdots \frac{b c}{c-b+a / q^{n}}+\cdots \cdot
$$

Proof. Changing $\lambda$ to $0, a$ to $-b / a q$ and $b$ to $c / a$ in (II) $=(\mathrm{IV})_{\mathrm{R}}$ and taking reciprocal we have

$$
\begin{aligned}
& \frac{g(-b / a q, 0, c / a, q)}{g(-b / a, 0, c / a, q)} \\
& \quad=1+\frac{-b / a}{1+(b+c q) / a}+\cdots \frac{-b / a}{1+\left(b+c q^{n}\right) / a}+\cdots \\
& \quad=\left(1-\frac{b}{a}\right)+\frac{b c q / a^{2}}{1+q(c-b) / a}+\cdots \frac{b c q^{2 n-1} / a^{2}}{1+q^{n}(c-b) / a}+\cdots
\end{aligned}
$$

Multiplying (19) by $a$ throughout and adding $c$ throughout we have

$$
\begin{aligned}
& c+\frac{a g(-b / a g, 0, c / a, q)}{g(-b / a, 0, c / a, q)} \\
& \quad=a+c+\frac{-a b}{a+b+c q}+\cdots \frac{-a b}{a+b+c q^{n}}+\cdots \\
& =a+c-b+\frac{b c q}{a+(c-b) q}+\cdots \frac{b c q^{2 n-1}}{a+(c-b) q^{n}}+\cdots
\end{aligned}
$$

We complete the proof by taking the reciprocal of (20) throughout. In addition to proving $(5)_{R}$ we have thus obtained that each side of $(5)_{R}$ equals

$$
\left\{c+\frac{a g(-b / a q, 0, c / a, q)}{g(-b / a, 0, c / a, q)}\right\}^{-1} \text {. }
$$

\section{REFERENCES}

1. G. E. Andrews, An introduction to Ramanujan's “lost” notebook, Amer. Math. Monthly 86 (1979), 89-108.

2. __ Ramanujan's “lost" notebook. III: The Rogers-Ramanujan continued fraction, Adv. in Math. 41 (1981), 186-208.

3. M. D. Hirschhorn, A continued fraction, Duke Math. J. 41 (1974), 27-33.

4. , A continued fraction of Ramanujan, J. Austral. Math. Soc. Ser. A 29 (1980), 80-86.

Department of Postgraduate Studies and Research in Mathematics, University of Mysore, MANASAGANGOTRI, MYSORE - 570006, INDIA 